\title{
Study of Functional Materials by Correlative Electron and Synchrotron X-ray Microscopy
}

\author{
Yuzi Liu, Tao Zhou, Xinwei Zhou, Zhonghou Cai and Martin Holt
}

Argonne National Laboratory, United States

Electron and synchrotron X-ray microscopy with wide range of spatiotemporal resolution, advanced detectors and data science enable the study of emerging science in condensed matter physics, chemistry and material science. In-situ/operando electron microscopy has been employed to study the energy materials transformation from atomic to mesoscopic scale in structure and chemistry.,$\underline{2}$ Synchrotron Xray technology with great brightness and coherence offers enormous opportunities for in-situ characterization techniques in imaging, scattering and spectroscopy to study the structural and chemical evolution of functional materials in operating environments at great accuracy in real time $\frac{3-5}{\text {. Integrated }}$ correlative electron and X-ray microscopy for complementary analysis of materials transformation provides the insights in multi-modalities at different length and time scales to reveal the working mechanism of functional materials like catalysis. $\underline{6}$ Data science and artificial intelligence algorithm speeds up the data analysis with improved accuracy and cross-correlate the findings from multimodal characterizations to accelerate science discovery.

The characterization results from one platform can help to identify the best sample location and potential science to be studied by another characterization method. For example, the scanning X-ray nanospectroscopy result from hard X-ray nanoprobe was the guidance for electron energy loss spectroscopy study from TEM at high spatial resolution to find active sites for $\mathrm{CO}_{2}$ reduction by $\mathrm{Cu}_{2} \mathrm{O}^{6} \cdot \mathrm{Such}$ multimodal characterization also cross verified the true science of the materials which might be compromised by experimental artifacts from instrumentation or beam effect which happens very often in beam sensitive materials. In particular, beam sensitive materials can benefit from multimodal characterizations. Study of the 2D materials needs both sensitivity and accuracy due to the weak signals from the very limited volume of materials in direction of thickness. Correlative electron and X-ray imaging and scattering provide the deep structural information about the strain distribution at sub-nanometer resolution by geometry phase analysis based on high resolution electron microscopy image and scale of strain extension in lateral dimensions from scanning X-ray nanoprobe scattering.

To bring various imaging modalities to bear on the same research object, some challenges should be addressed. Considering the disparate length scales between different microscopy techniques, uncertainties in the sample should be quantified. A shareable sample holder is important to enable the identical samples to be investigated across the correlated electron and X-ray microscopy platforms.

This work was performed at the Center for Nanoscale Materials, a U.S. Department of Energy Office of Science User Facility, and supported by the U.S. Department of Energy, Office of Science, under Contract No. DE-AC02-06CH11357. 

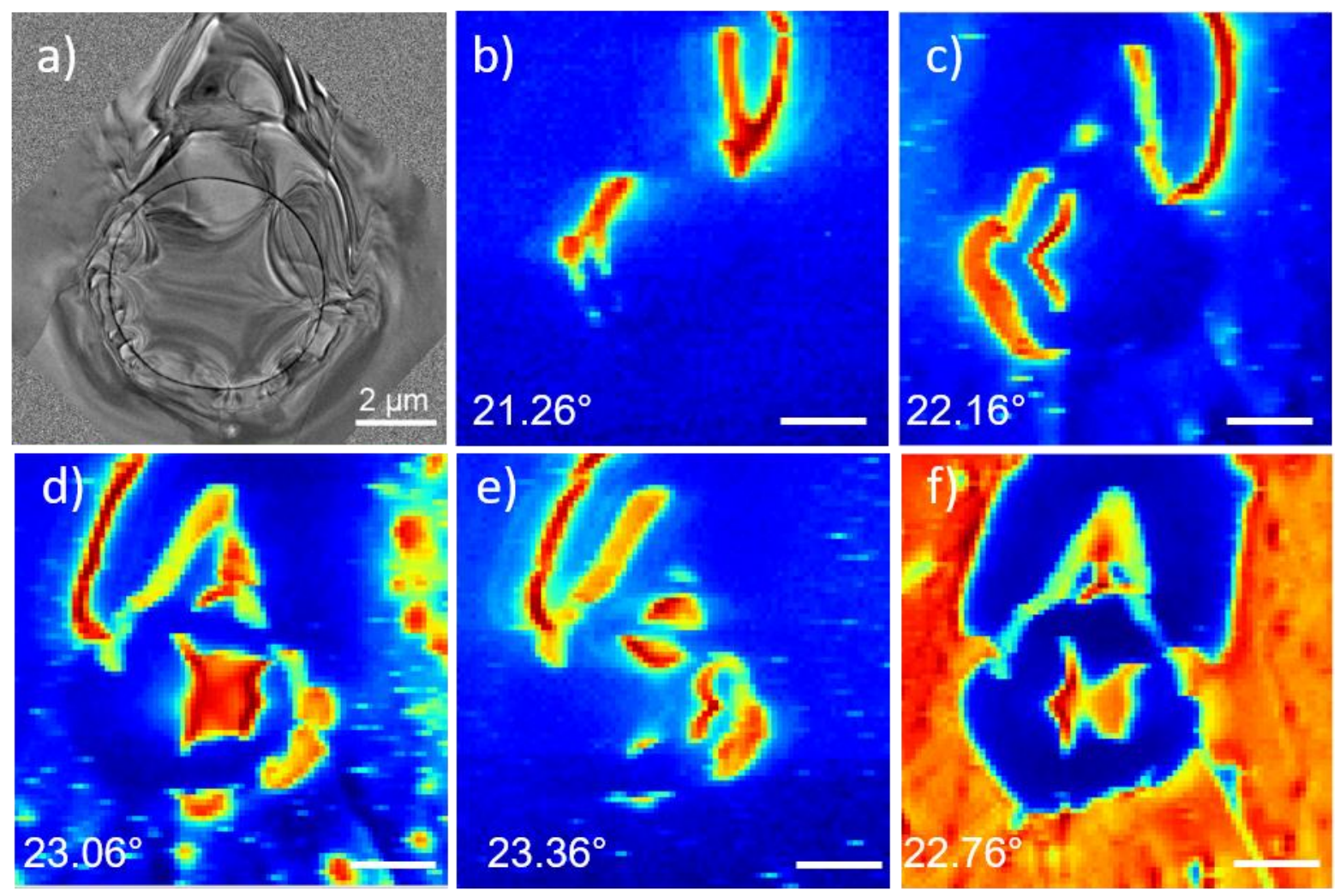

Figure 1. The suspend WSe2 on $\mathrm{SiN}$ membrane with open hole (dark circle). a) bright field TEM micrograph shows the wrinkled WSe 2 which was prepared by standard exfoliation process. b)-f) show the integrated diffraction intensity from scanning nanodiffraction at 26ID at Advanced Photon Source at Argonne National Laboratory. The number in each figure is the scattering angle. The results tell the lattice orientation change along with the wrinkles on the sample. The scale bars are $2 \mu \mathrm{m}$.

(a)
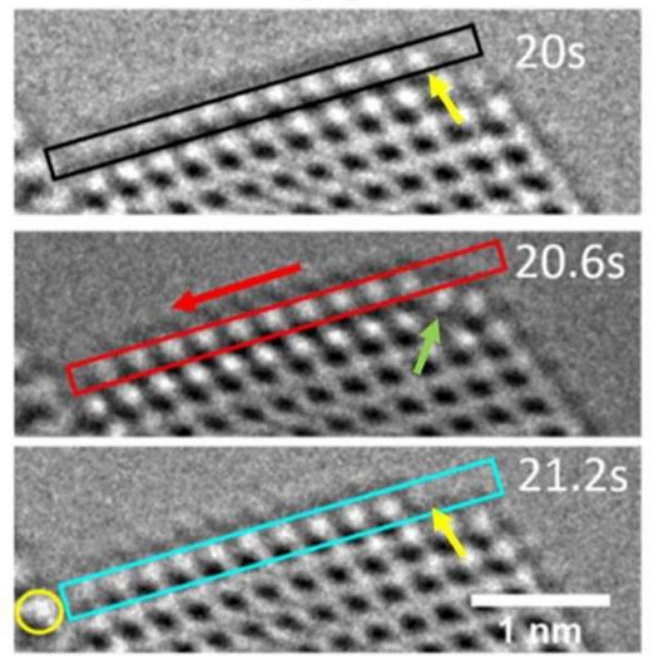

(b)

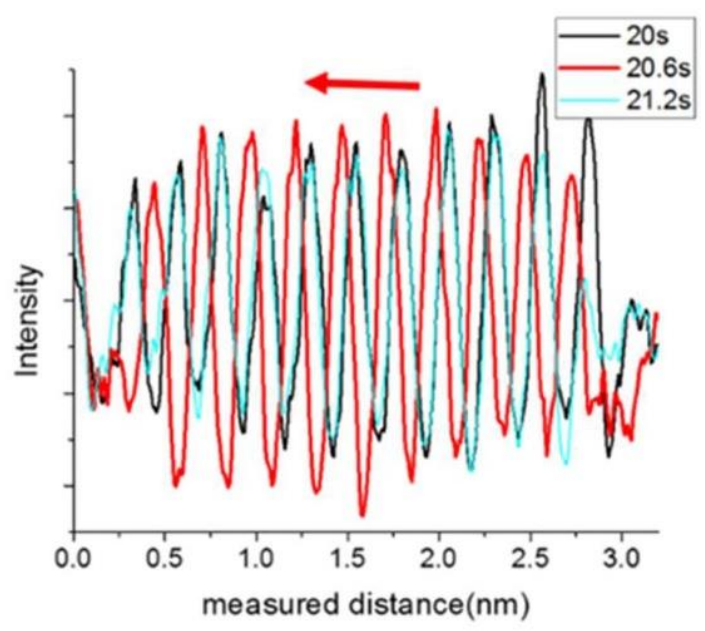




\section{References}

1. Liu, X. H.; Wang, J. W.; Huang, S.; Fan, F.; Huang, X.; Liu, Y.; Krylyuk, S.; Yoo, J.; Dayeh, S. A.; Davydov, A. V.; Mao, S. X.; Picraux, S. T.; Zhang, S.; Li, J.; Zhu, T.; Huang, J. Y., In situ atomicscale imaging of electrochemical lithiation in silicon. Nature Nanotechnology 2012, 7 (11), 749-756.

2. Zhou, X. W.; Li, T. Y.; Cui, Y.; Meyerson, M. L.; Weeks, J. A.; Mullins, C. B.; Jin, Y.; Liu, Y. Z.; Zhu, L. K., In Situ and Operando Morphology Study of Germanium-Selenium Alloy Anode for Lithium-Ion Batteries. Acs Applied Energy Materials 2020, 3 (7), 6115-6120.

3. Ulvestad, A.; Singer, A.; Clark, J. N.; Cho, H. M.; Kim, J. W.; Harder, R.; Maser, J.; Meng, Y. S.; Shpyrko, O. G., Topological defect dynamics in operando battery nanoparticles. Science 2015, 348 (6241), 1344-1347.

4. Whiteley, S. J.; Heremans, F. J.; Wolfowicz, G.; Awschalom, D. D.; Holt, M. V., Correlating dynamic strain and photoluminescence of solid-state defects with stroboscopic x-ray diffraction microscopy. Nature Communications 2019, 10 (1), 3386.

5. Holt, M. V.; Hruszkewycz, S. O.; Murray, C. E.; Holt, J. R.; Paskiewicz, D. M.; Fuoss, P. H., Strain Imaging of Nanoscale Semiconductor Heterostructures with X-Ray Bragg Projection Ptychography. Phys. Rev. Lett. 2014, 112 (16), 165502.

6. $\quad$ Wu, Y. A.; McNulty, I.; Liu, C.; Lau, K. C.; Liu, Q.; Paulikas, A. P.; Sun, C. J.; Cai, Z. H.; Guest, J. R.; Ren, Y.; Stamenkovic, V.; Curtiss, L. A.; Liu, Y. Z.; Rajh, T., Facet-dependent active sites of a single $\mathrm{Cu} 2 \mathrm{O}$ particle photocatalyst for $\mathrm{CO} 2$ reduction to methanol. Nature Energy 2019, 4 (11), 957968. 